\title{
A Method for Voltage Noise Measurement and Its Application to Primary Batteries
}

Can Berk Uzundal* and Burak Ulgut $\oplus^{* *, z}$

Department of Chemistry, Bilkent University, 06800 Ankara, Turkey

\begin{abstract}
Though proven effective in localized corrosion studies, electrochemical noise measurements in batteries with Lithium based chemistries suffer from lack of well-defined measurement and analysis methods. The high capacitance electrodes made out of highly porous materials requires noise measurements to be extremely precise since the small charge due to stochastic events leading to electrochemical noise leads to very small voltage changes due to the large capacitance. Typically, the required precision is achieved by high gain after the offset is corrected. In this article, we are introducing a new offset correction scheme that mitigates the negative effects of electronic offset reduction methods. Using this new offset correction scheme we report the measurement of the otherwise elusive voltage noise of primary Li batteries.

(C) The Author(s) 2018. Published by ECS. This is an open access article distributed under the terms of the Creative Commons Attribution 4.0 License (CC BY, http://creativecommons.org/licenses/by/4.0/), which permits unrestricted reuse of the work in any medium, provided the original work is properly cited. [DOI: $10.1149 / 2.0681811 \mathrm{jes}$ ]

(cc) BY
\end{abstract}

Manuscript submitted June 18, 2018; revised manuscript received July 27, 2018. Published August 21, 2018.

Information regarding the underlying processes that occur during the normal operation of electrochemical energy storage and conversion systems can be extracted through processing signals that are either the results of applied external excitations or intrinsically generated. These techniques elucidate the underlying processes to both diagnose the state of the systems and also gain insights to increase next generation performances. ${ }^{1,2}$ Electrochemical noise measurements with the appropriate measurement and signal processing routines are expected to be relevant in this regard where electrochemical noise can be a vital indicator of the underlying processes that occur even at an apparent equilibrium. Especially for primary chemistries that are inherently not rechargeable, nondestructive/equilibrium techniques such as electrochemical noise is attractive where conventional techniques deplete the battery in question during the quality control process. For various other attempts at non-destructive battery quality control methods see the following References 3, 4. Electrochemical Impedance Spectroscopy (EIS) is the method of choice for battery quality control. ${ }^{5}$ It is usually a non-invasive technique owing to the small and symmetric excitations employed. For primary chemistries, however, excitations need to have a DC offset such that the battery is always discharged. After multiple EIS measurements, significant discharge occurs, especially for small cells. Therefore, EIS becomes an invasive measurement whereas noise measurements, since they are done at the equilibrated state without any external excitations, are perfectly non-invasive.

Though underappreciated in academic circles, the market share of the primary batteries are still expected to continuously grow in the near future and thus primary battery diagnosis is still an open question. ${ }^{6}$ Primary chemistries are prevalently used in household items and medical tools due to their high and steady energy outputs in addition to their military uses.

The electrochemical noise literature is rich with both well-defined measurement systems and data analysis methods. Application of these methods to corrosion systems were thoroughly reviewed by Cottis. ${ }^{7}$ Further, a guideline to standardize electrochemical noise in studies of corrosion were published by ECG-COMON. ${ }^{8}$ If measured properly, even local behavior can be extracted from monitoring voltage or current noise of corroding systems. ${ }^{9-11}$ Recently, electrochemical noise measurement of fuel cells and batteries have been attracting interest as a diagnostic tool due to the non-invasive nature of the measurement. Residual water content of the fuel cell, which is known to be detrimental, was shown to correlate with the electrochemical noise of the system. ${ }^{12}$ On another example, calendar aging of supercapacitors were followed by careful investigations of their flicker

\footnotetext{
*Electrochemical Society Student Member.

***Eectrochemical Society Member.

ż-mail: ulgut@fen.bilkent.edu.tr
}

noise. ${ }^{13}$ In batteries, early reports of electrochemical noise measurements were conducted using aqueous chemistries as extensions to the corrosion studies. Lead-acid batteries were the focus of these reports where electrolysis can be followed via noise measurements. ${ }^{14}$ Similar measurements were further extended to $\mathrm{Ni}$ based aqueous systems as well. ${ }^{15,16}$ Early studies of noise in Lithium chemistries were conducted under galvanostatic conditions where gas evolution is followed via noise measurements. ${ }^{15}$ Yet, noise measurements involving new generation batteries, especially Li-Ion batteries at the equilibrated state, are sparse in the literature. Only recently, articles measuring voltage noise of commercially available Li-Ion batteries under open circuit condition with very low backgrounds was published. ${ }^{17}$ The conclusions drawn from this low background electrochemical noise data, however, is highly suspect since the published analysis suffers from lack of appropriate de-trending. The need for well-defined analysis methods and measurement techniques is clear. The apparent noise should be, unequivocally, from the battery while the numerical handling of the stochastic data should not introduce artifacts.

Though both voltage and current noise are routinely measured in corrosion studies, current noise measurements of batteries are dominated by the noise introduced to the system due to the noise in the applied potential. Therefore, noise studies on batteries are focused on voltage noise only. ${ }^{13-15,17}$ For simplicity, throughout the rest of this manuscript, noise always refers to voltage noise.

In the literature, to draw both quantitative and qualitative conclusions from noise data, usually a background measurement is employed that involves a shorted lead measurement and resistors of relevant resistance levels. ${ }^{7}$ Although resistor experiments can guide the initial inquiry, voltage noise of batteries are affected by issues that are not reflected in resistor measurements.

An analog process that may introduce artifacts to the measured signal at amplitudes relevant to noise measurements $(\sim \mu \mathrm{V})$ is offset subtraction. Most instruments achieve high resolution even at high voltage amplitudes by ranging and offset subtraction as illustrated in Figure 1. The smallest voltage that can be measured above the quantization error is determined both by the amplitude of the voltage signal to be converted and the resolution of the A/D converter (given by the number of bits available). Roughly, offset correction subtracts the static part of the voltage from the whole thus reducing the voltage amplitude and increasing the resolution on the analog-to-digital converter. Our experiments show that this offset correction may significantly contribute to the apparent noise and can be circumvented by using another battery of the same voltage in an anti-serial connection (i.e. connected in series with same sign poles connected to each other) as shown in Figure 2. When two batteries are attached in parallel, they are at a high voltage amplitude $(4.1 \mathrm{~V})$ which requires offset correction to achieve high resolution. During this offset correction noise that 


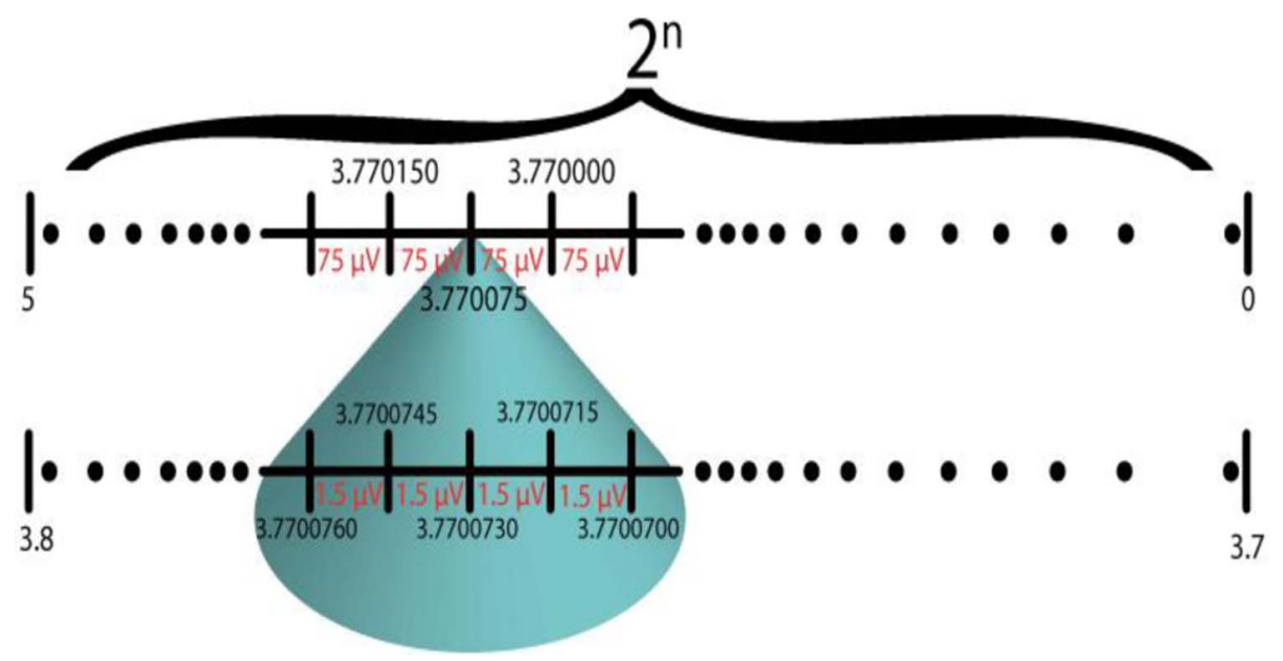

Figure 1. Illustration of the increased resolution by offset reduction.

stems from the instrument is introduced to the measurement. In the anti-serial connection, the voltage that needs to be measured by the instrument is effectively already offset corrected (it is at $0 \mathrm{~V}$ ) which enables the high resolution required for noise measurements. Offset correction by anti-serial connection achieves the high resolution required for battery noise measurements while mitigating the noise introduced by the electronic offset reduction.

\section{Materials and Methods}

SANYO 18650 Li-Ion batteries of 3400mAh capacity, MAXELL CR2032 (batch 63) coin-cells and 5F Nesscap Supercapacitors were procured from local suppliers. Supercapacitors and the 18650 batteries were charged to the desired voltage and left to relax over a day such that voltage equilibration is complete (voltage changes less than $1 \mathrm{mV} / \mathrm{min}$ ) prior to noise measurement.

Impedance of primary batteries were measured galvanostatically with a DC offset of $-2 \mu \mathrm{A}$ and $\mathrm{AC}$ amplitude of $2 \mu \mathrm{A}$ in the frequency region of $10 \mathrm{mHz}$ to $50 \mathrm{kHz}$.
All measurements were done inside an earth grounded Faraday's cage to ensure the quality of the experiment. The energy storage devices were connected to the Gamry Interface 5000 in a two electrode configuration. ESA 410 software which specializes in measurement of electrochemical noise was utilized. Using this software, the potentiostat can be used as a galvanostat with an output frequency of $500 \mathrm{~Hz}$ and an output filter of $0.1 \%$. The data was sampled at $500 \mathrm{~Hz}$. Only for primary batteries the output frequency and the sampling rate was lowered guided by our initial experiments on primary batteries where the battery noise was mostly seen in the low frequency region. For these systems, the potentiostat was operated as a galvanostat with an output frequency of $5 \mathrm{~Hz}$, sampled at $5 \mathrm{~Hz}$.

The voltage noise measured has a contribution from the slow selfdischarge of the batteries and various instrumental offsets. The analytical form of self-discharge can be extremely complex and may not be known ${ }^{18}$ therefore directly removing it from the voltage noise with a non-linear fit is not viable. Instead a piecewise linear de-trend is used in the literature to remove this background. ${ }^{7}$ The obtained detrended voltage can be taken to the frequency domain and analyzed,

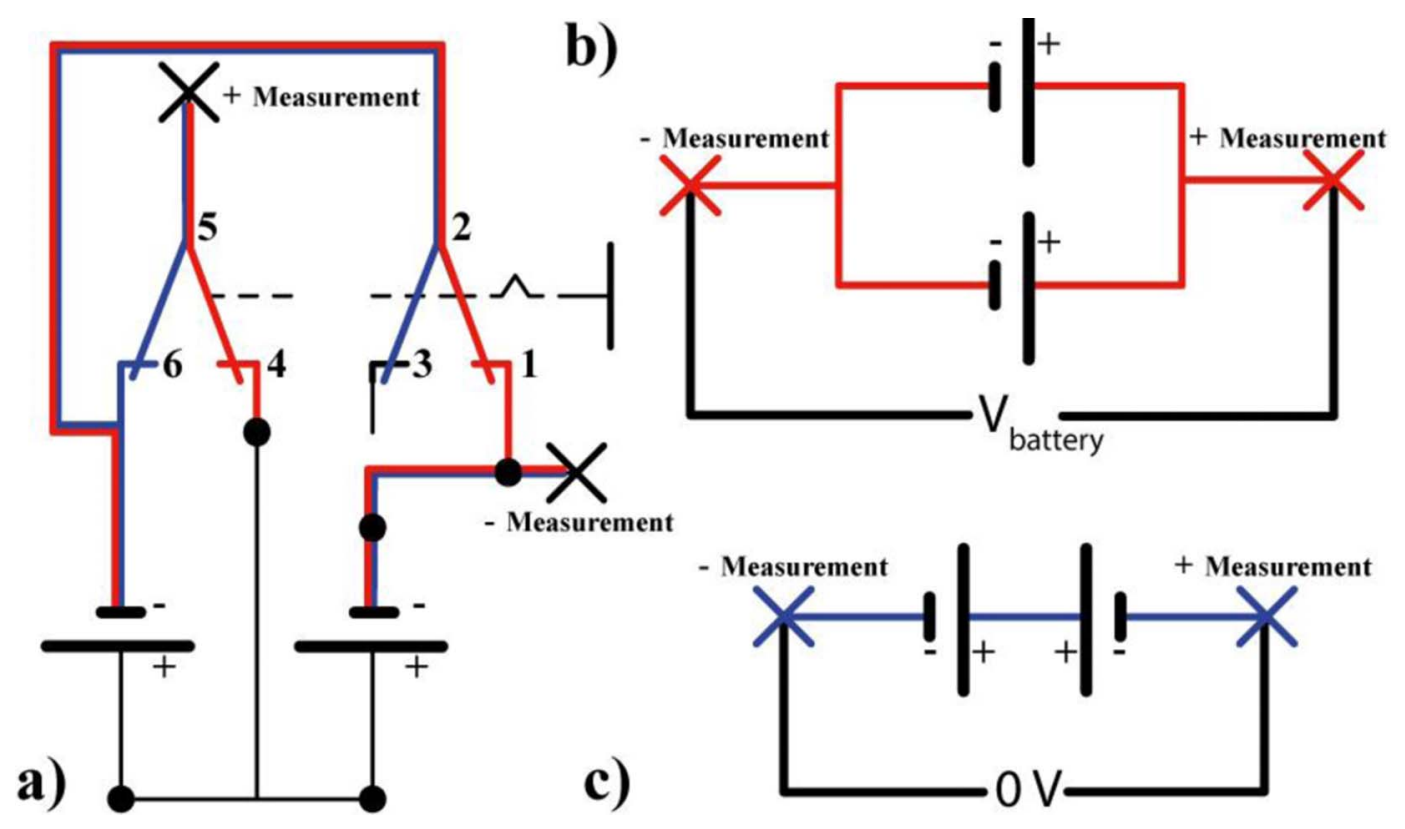

Figure 2. a) Sliding switch that changes connections from parallel (red) to anti-serial (blue). b) When the connections 1-2 and 5-4 are selected, the batteries are in parallel as shown. c) When the connections 3-2 and 6-5 are selected, the batteries are in anti-serial as shown. 

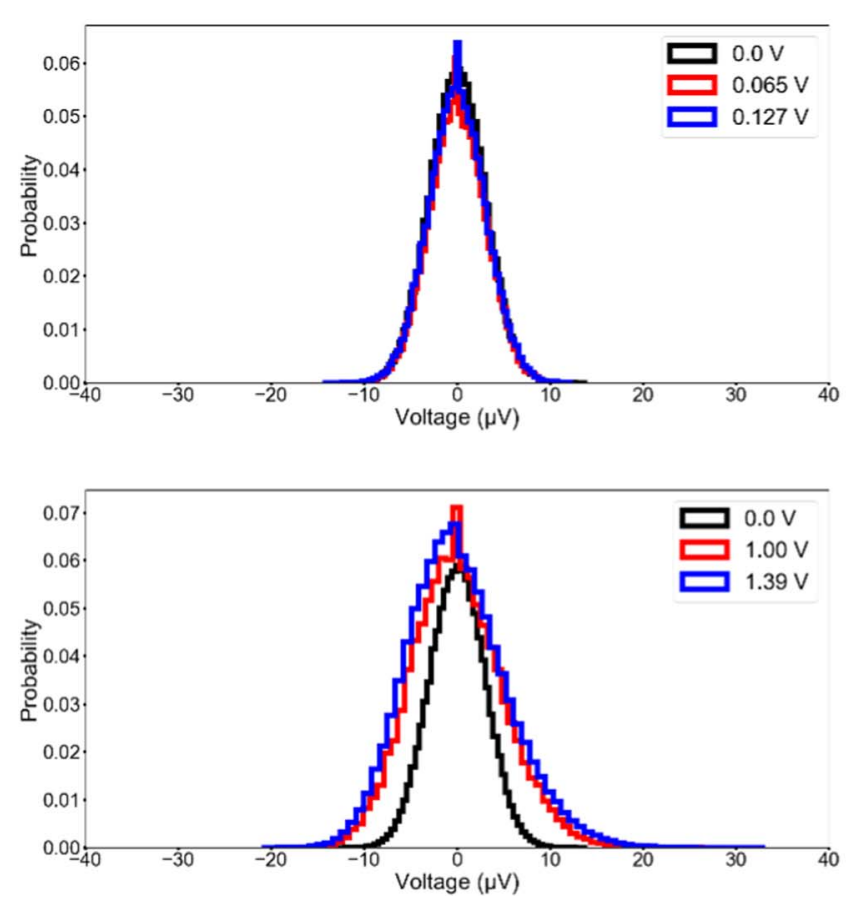
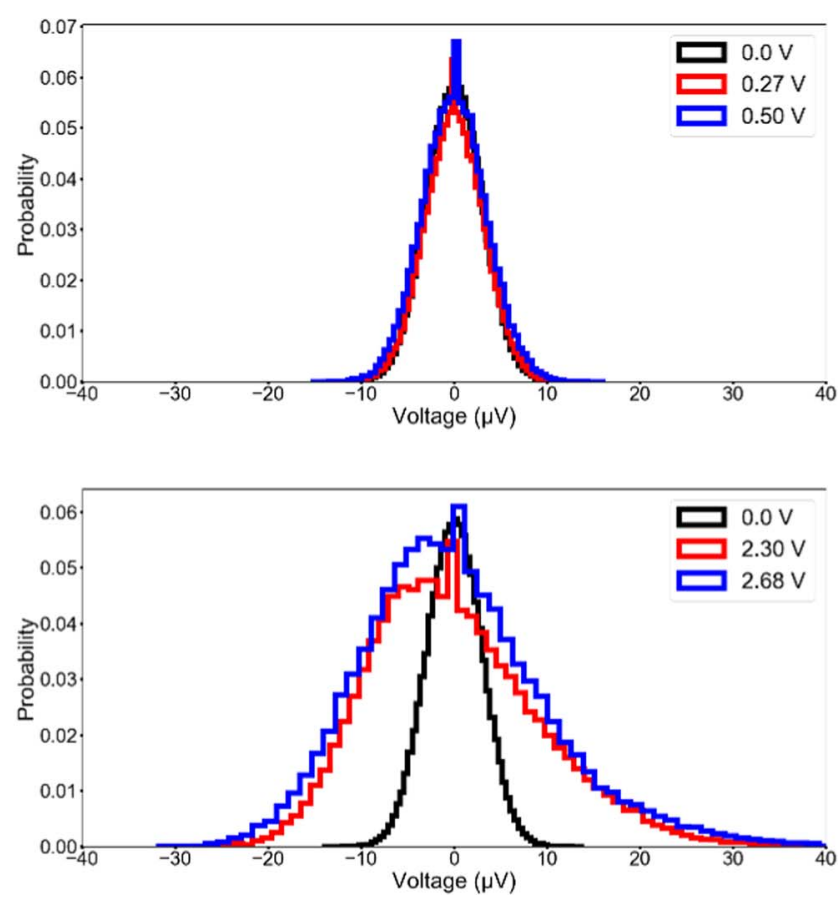

Figure 3. Apparent noise of a 5F Supercapacitor at different states-of-charge. Time traces and their Fourier transforms (FFT) can be found in Figure S1.

however the voltage trace is not periodic but rather stochastic, thus limited insight is gained from the f-domain traces. Electrochemical noise literature is rich with reports that highlight methods that prefer time domain analyses. Wavelet transforms capture finer details by applying non-periodic signals. ${ }^{19,20}$ Recurrence Quantification Analysis shows the degree of repetition and correlation in the otherwise stochastic data ${ }^{11,21}$ and Stochastic Process Detection (SPD) algorithm developed by Roberge can catalog stochastic events in terms of their shape to quantify trends. ${ }^{22}$ For the purposes of this article, a plainer approach was taken and time traces were visualized using histograms.

Noise measured was first linearly de-trended by dividing the whole voltage trace into 1024-point chunks and individually subtracting the best-fit line from each chunk. This piecewise linear de-trended voltage is then either visualized using histograms of 64 bins or taken to the frequency domain using FFT and visualized.

All data visualizations and array operations were done in Python 2.7.10.0 using Matplotlib, ${ }^{23}$ Numpy $^{24}$ and Scipy under a Python(x,y) shell.

\section{Results and Discussion}

Apparent noise is a superposition of the actual noise of the battery and the noise introduced by the measurement instrument. Noise of the battery could be stemming from either faradaic or non-faradaic processes. In contrast to the earlier reports involving the noise of aqueous chemistries, electrolysis (faradaic contribution) is not a significant factor in Li-Ion batteries and therefore the amount of noise is not as large. This puts the level of noise in an order of magnitude where most of the noise that occur inside the battery should be shunted by the high capacitance of the electrodes. ${ }^{25}$ To distinguish between these two distinct noise sources, supercapacitors are a valuable tool, since their charge storage mechanism is purely non-faradaic. If employed at the same voltage and with electrodes of similar capacitance as the electrodes of the battery in question, a supercapacitor is an ideal control sample for battery noise. The background provided by supercapacitor noise not only can distinguish between faradaic and non-faradaic sources of battery noise but also can show whether the apparent noise is an instrumental artifact.

Further, supercapacitors allow noise measurements at voltages that are not available within the operational window of a Li-Ion battery.
Figure 3 (see Figure S1 for time domain and frequency domain traces) shows the apparent noise of a $5 \mathrm{~F}$ supercapacitor measured at different states-of-charge. The normalized histograms centered around $0 \mathrm{~V}$ widen with the increasing voltage of the supercapacitor, indicating an increase in the apparent noise. Similar experiments with batteries are much less conclusive as the available voltage window is much narrower.

If the observed increase in apparent noise is indeed due to the state of charge and not an instrumental artifact, apparent noise should show a similar behavior regardless of the average voltage measured by the instrument as long as the state of charge of the battery or the supercapacitor is preserved. Therefore, attaching another battery or supercapacitor of the same voltage (i.e. state of charge) in an anti-serial connection should yield similar apparent noise profiles. However, the apparent noise at these voltages when two supercapacitors are connected in an anti-serial connection yields an apparent noise that is similar to the shorted lead of the instrument. Although this shows that the apparent noise is an instrumental artifact at these voltage amplitudes, the possibility of using supercapacitors as a background measurement for voltage noise and the higher voltage sensitivity achieved by the anti-serial connection needs to be highlighted. In this article, we leverage the enhanced voltage resolution enabled by the anti-serial connection to measure the noise of various energy storage devices (such as supercapacitors, Li \& Li-Ion batteries). The objective of this study is two-fold. We outline the factors that need to be handled for proper noise measurements for these systems with low stochastic noise and a high offset voltage (supercapacitors \& LiIon batteries) and use the outlined methodology to demonstrate the potential of noise measurements as a non-destructive quality control tool for primary Li batteries.

In order to accurately offset the DC voltage, the two cells to be connected anti-serially have to be at precisely the same potential. Therefore, the best experimental workflow is to keep the cells connected in parallel to force the potentials to be the same, let them equilibrate to the same potential and then switch to the anti-serial connection for the noise measurement without any need for an electronic offset correction. Therefore, a simple switch was designed to alternate between parallel and anti-serial connections as shown in Figure 2. This ability to quickly alternate between parallel and anti-serial connections helps with equilibrating the two batteries to the same potential using the 


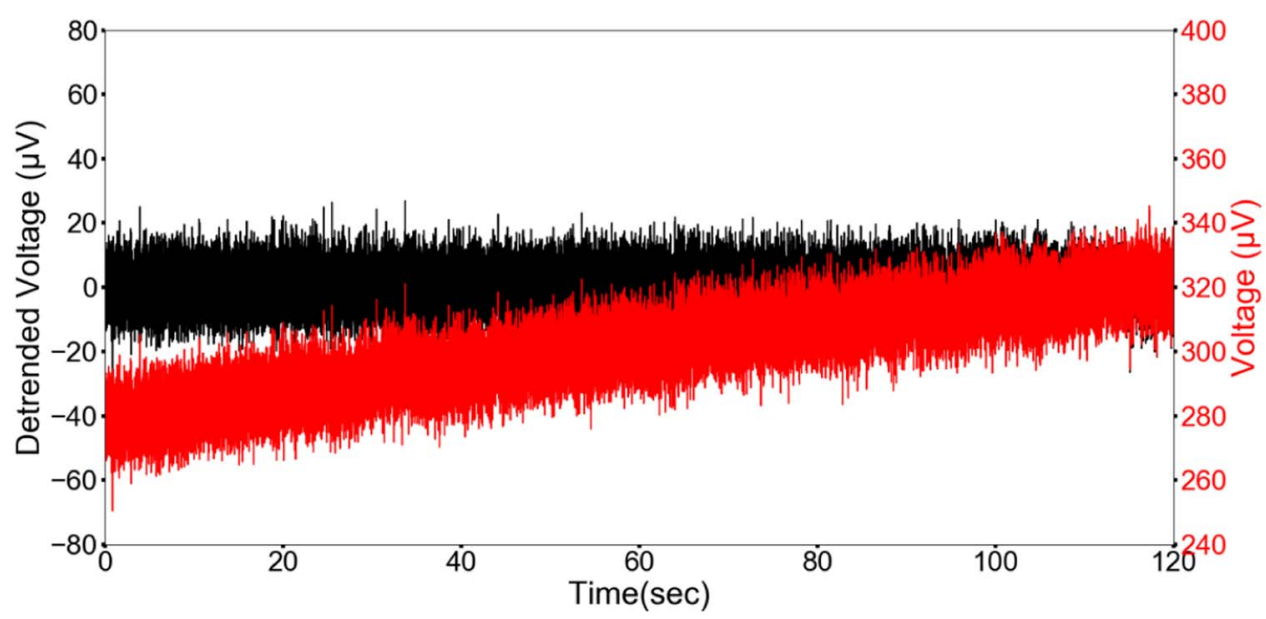

Figure 4. Apparent noise at $4.1 \mathrm{~V}$, de-trended time trace shown in black and the pristine trace is shown in red.

parallel connection and quickly switching to the anti-serial position to achieve zero overall potential.

18650 Li-Ion battery noise.-In a case where the apparent noise is purely of the battery, the anti-serial connection would show noise levels similar to or exceeding the single battery measurement. This was not seen for the noise of $18650 \mathrm{Li}$-Ion batteries charged to $4.1 \mathrm{~V}$. Apparent noise of two 18650 batteries connected in a parallel connection reduces to the same level as the shorted lead noise of the instrument when attached in an anti-serial connection (Figure S2). The apparent noise of the parallel/high amplitude case being significantly higher, while, the apparent noise of the anti-serial connection being the same as the shorted lead, proves that the apparent noise is dominated by instrumental artifacts at high voltage amplitudes. Considering that the large capacitance of the high surface area electrodes damp the effects of events on electrodes, ${ }^{25}$ instrumental factors that contribute to the apparent noise should be fully accounted for. Among these instrumental factors the input bias and the oscillations of the electrometer in the frequency region of interest should be minimized. For a given instrument changing these factors is, in most cases, not possible (see the discussion titled Comparison of Various Instruments in the Supplementary Material).

De-trending.-Using the anti-serial offsetting scheme outlined in this article does not fully mitigate the need to de-trend the apparent noise, where small drifts in voltage can still occur. Commercial batteries of the same manufacturer, even from the same batch, have slight differences in terms of their impedance and capacity. ${ }^{26}$ This alone can cause a slight drift, which should be de-trended as shown in Figure 4. If the measured noise is not de-trended, it is easy to draw ill-guided conclusions influenced by the statistics of a should-be stationary system applied to a non-stationary one. Especially noise power analysis and high order statistics are known to be highly susceptible to drift. ${ }^{7}$

Primary lithium battery noise.-Discussions outlined in the above sections (and in the Supplementary Material) form a basis for the proper measurement of battery noise. As discussed, the large capacitance of the $18650 \mathrm{Li}$-Ion batteries lowers the noise amplitude and render them practically unmeasurable with our instruments. Thus smaller batteries are expected to be more amenable for noise measurements. As such, we selected CR2032 coin-cells and measured their noise. Another added benefit of this chemistry is their relatively high internal resistance which improves the electrometer stability (also see the discussion titled Electrometer Stability in the Supplementary Material). Further, in order to mitigate the adverse effects of the high frequency noise components in time domain analyses, a narrower bandwidth of $2.5 \mathrm{~Hz}$ is chosen in these measurements.

The apparent noise at $3.2 \mathrm{~V}$ without applying the anti-serial connection outlined in this article shows a noise signal that vanishes when attached in the anti-serial connection. The disappearance of the

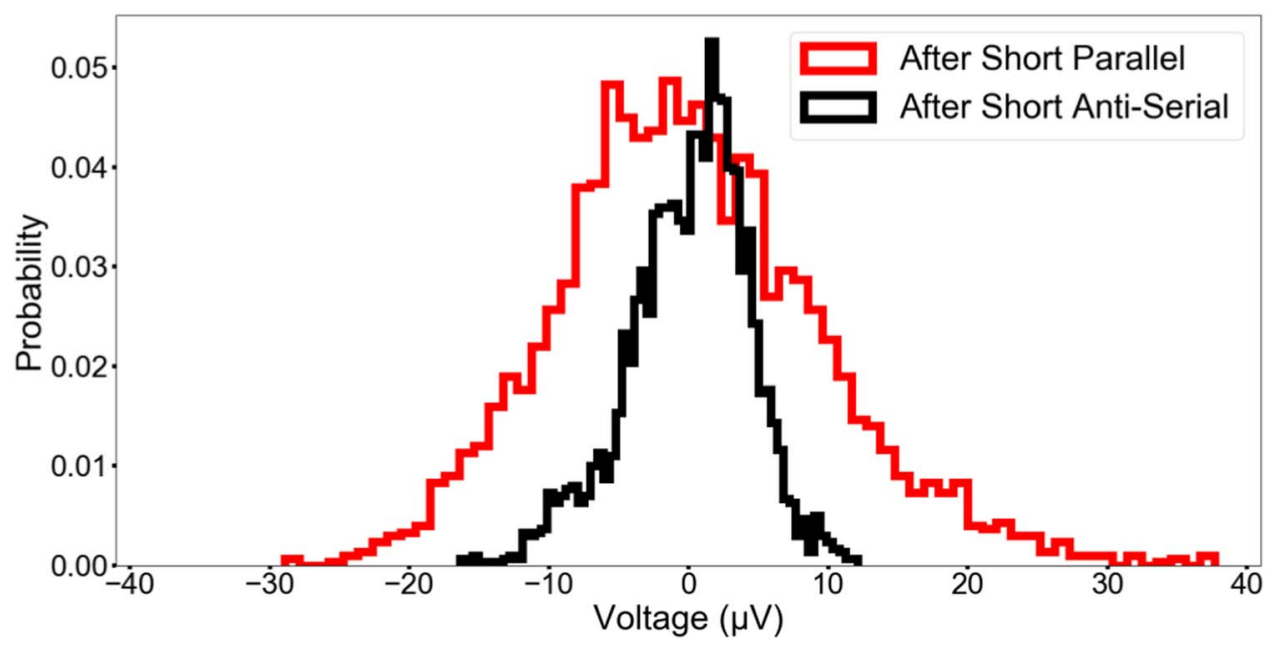

Figure 5. Noise of the same two CR2032 batteries compared in anti-serial and parallel connections after short circuiting the batteries. 

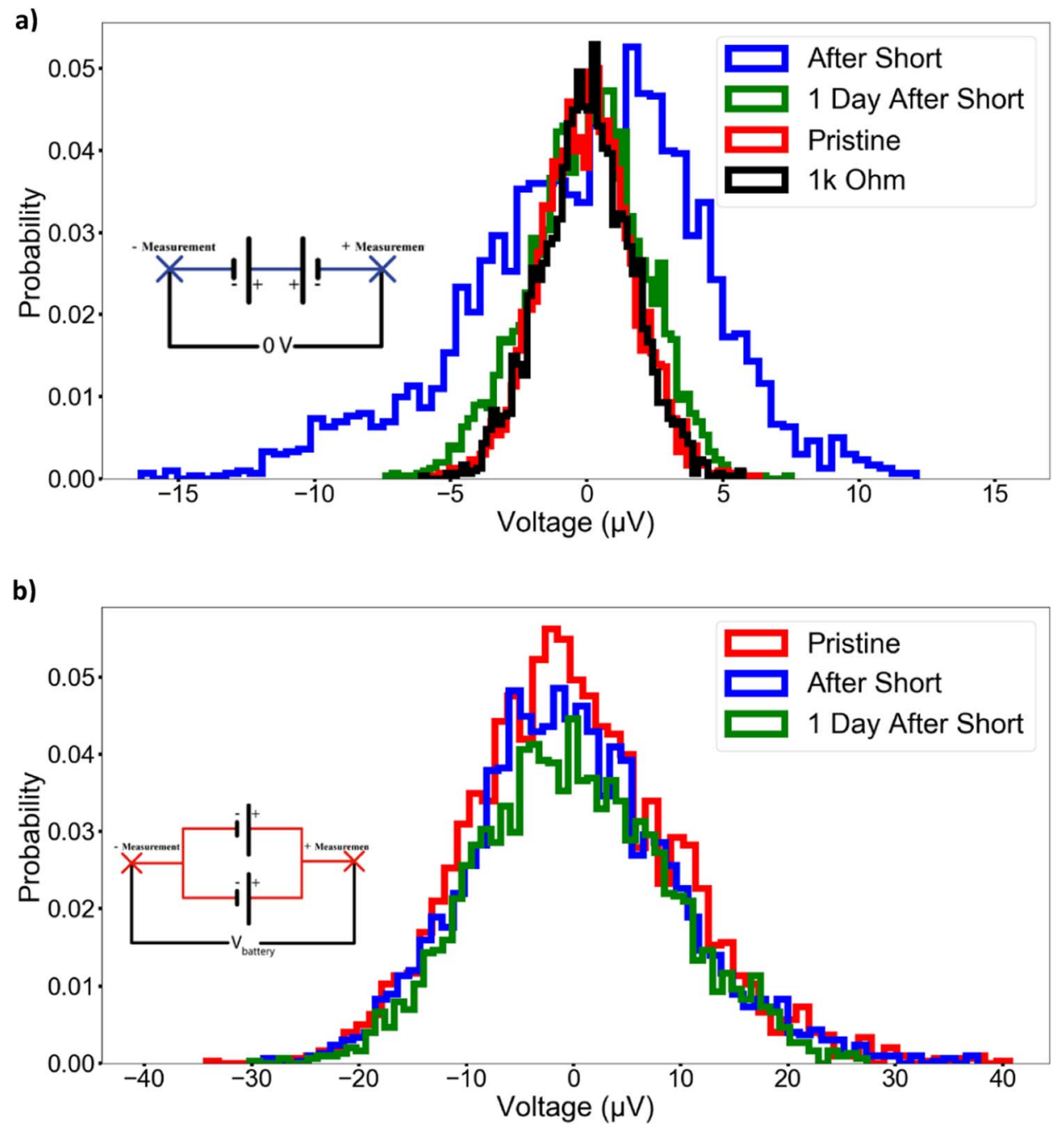

Figure 6. a) Noise of two CR2032 coin-cells attached in anti-serial connection. b) Noise of the same two CR2032 coin-cells attached in parallel connection. (Figure 5 serves as a scale for these two graphs for ease of view).

noise signature to the shorted lead levels in the anti-serial connection again confirms that the noise measured at the high amplitude is an instrumental artifact introduced by the offset correction scheme employed by the instrument. The increased voltage resolution of the antiserial case is evident when comparing the apparent noise histograms obtained under same conditions for the parallel and anti-serial connections as shown in Figure 5. Individually shorting these batteries overnight and measuring the noise once they settle, however, yields a remarkable increase in the measured noise (Figure 6a). An appropri- ate background for the anti-serial connected batteries is the apparent noise measured over a resistor of comparable resistance. For the batteries after shorting, the internal resistance increases significantly and approaches around $1 \mathrm{k} \Omega$ (see Figure S3). This background is not appropriate for the noise measurements in the parallel connection due to the above discussed offset correction artifacts introduced by the instrument to the measurement in question. Instead the noise of the pristine battery can be taken as the apparent noise floor. Clearly after short circuiting the batteries, the apparent noise rises above the a)

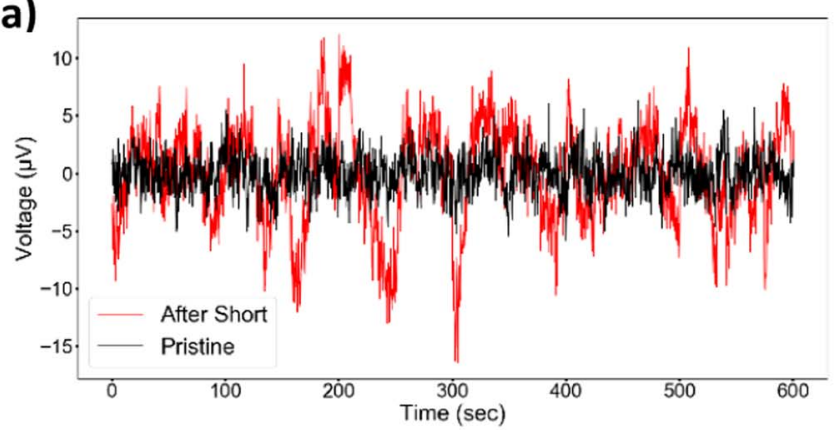

b)

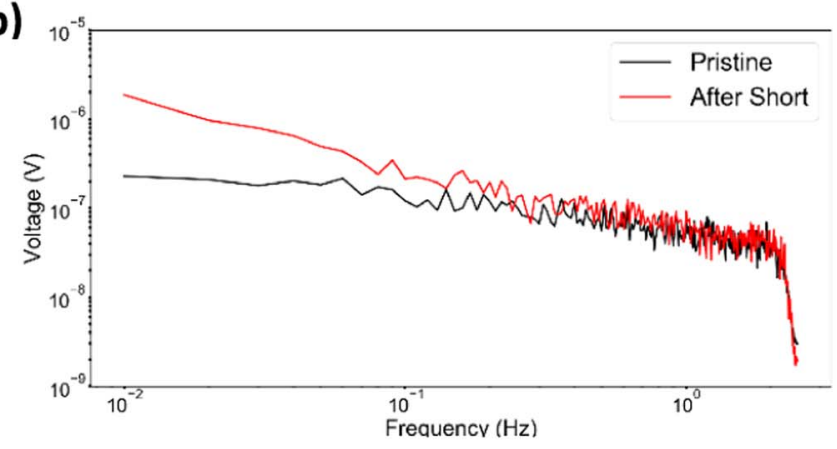

Figure 7. Noise of two CR2032 coin-cells attached in anti-serial connection a) in the time domain. b) in the frequency domain. 
noise floor for the anti-serial connection while for the parallel connection no such increase can be discerned (see Figure S4 and S5 for the time domain and frequency domain responses). The inability of the parallel connection to catch the noise signature of the battery is expected especially when we compare the voltage amplitudes at which the battery noise occurs as shown in Figure 5 (around 4 times lower than the smallest voltage measurable in the parallel case). When the external short circuit is lifted, the coin-cell recovers its voltage over time (as confirmed by the impedance and the voltages of the cells, see Figure S3). The origins of this noisy recovery is currently under investigation. Clearly the obtained noise profile has information regarding the processes taking place in the battery similar to the corroding systems (Figure 7a), however the stochastic nature of the data, as seen by the frequency domain responses, complicate the quantification (Figure $7 b$ ) and consequently identification of these processes. The need for reliable time domain analysis methods is again stressed for proper quantification of these stochastic processes.

\section{Conclusions}

Offset correction by anti-serial batteries proved to be an effective approach in differentiating between the apparent noise and the actual noise of the system. The instruments available were pushed to their limits and improved upon by the new offset reduction scheme. Although the battery noise of the large Li-Ion batteries remains elusive and not detectable within the detection limit of our instruments, electrochemical noise of smaller coin-cells was detectable when shorted.

\section{ORCID}

Burak Ulgut (1D https://orcid.org/0000-0002-4402-0033

\section{References}

1. C. A. Little, R. Xie, C. Batchelor-McAUley, E. Katelhon, X. Li, N. P. Young, and R. G. Compton, "A Quantitative Methodology for the Study of Particle-Electrode Impacts," Phys. Chem. Chem. Phys., 13537 (2018).

2. A. R. Zeradjanin, E. Ventosa, A. S. Bondarenko, and W. Schuhmann, "Evaluation of the catalytic performance of gas-evolving electrodes using local electrochemical noise measurements," ChemSusChem. 5, 1905 (2012).

3. S. M. Lambert, M. Armstrong, P. S. Attidekou, P. A. Christensen, J. D. Widmer, C. Wang, and K. Scott, "Rapid nondestructive-testing technique for in-line quality control of li-ion batteries," IEEE Trans. Ind. Electron., 64, 4017 (2017).

4. M. Petzl and M. A. Danzer, "Nondestructive detection, characterization, and quantification of lithium plating in commercial lithium-ion batteries," J. Power Sources., 254, 80 (2014).
5. T. Hang, D. Mukoyama, H. Nara, N. Takami, T. Momma, and T. Osaka, "Electrochemical impedance spectroscopy analysis for lithium-ion battery using $\mathrm{Li}_{4} \mathrm{Ti}_{5} \mathrm{O}_{12}$ anode," J. Power Sources., 222, 442 (2013).

6. Technavio, Global Primary Battery Market 2018-2022, 2018. https://www. technavio.com/report/global-primary-battery-market-analysis-share-2018.

7. R. A. Cottis, "Interpretation of Electrochemical Noise Data," Corrosion. 57, 265 (2001).

8. S. Ritter, F. Huet, and R. A. Cottis, "Guideline for an assessment of electrochemical noise measurement devices," Mater. Corros., 63, 297 (2012).

9. G. Blanc, C. Gabrielli, and M. Keddam, "Measurement of the electrochemical noise by a cross correlation method," Electrochim. Acta., 20, 687 (1975).

10. K. Hladky and J. L. Dawson, "The measurement of localized corrosion using electrochemical noise," Corros. Sci., 21, 317 (1981).

11. W. Liu, D. Wang, X. Chen, C. Wang, and H. Liu, "Recurrence plot-based dynamic analysis on electrochemical noise of the evolutive corrosion process," Corros. Sci., 124, 93 (2017).

12. R. Maizia, A. Dib, A. Thomas, and S. Martemianov, "Proton exchange membrane fuel cell diagnosis by spectral characterization of the electrochemical noise," J. Power Sources., 342, 553 (2017).

13. A. Szewczyk, Lentka, J. Smulko, P. Babuchowska, and F. Béguin, Measurements of flicker noise in supercapacitor cells, 2017 Int. Conf. Noise Fluctuations, ICNF 2017. 2 (2017).

14. P. R. Roberge, R. Beaudoin, G. Verville, and J. Smit, "Voltage noise measurements on sealed leadacid batteries," J. Power Sources., 27, 177 (1989).

15. S. Martinet, R. Durand, P. Ozil, P. Leblanc, and P. Blanchard, "Application of electrochemical noise analysis to the study of batteries: state-of-charge determination and overcharge detection," J. Power Sources., 83, 93 (1999).

16. B. Ulgut, "Analysis of electrochemical noise in NiCd batteries throughout their lifetime," Turkish J. Chem., 1 (2018).

17. S. Martemianov, N. Adiutantov, Y. K. Evdokimov, L. Madier, F. Maillard, and A. Thomas, "New methodology of electrochemical noise analysis and applications for commercial Li-ion batteries," J. Solid State Electrochem., 19, 2803 (2015).

18. E. Ozdemir, (2017), Impedance Based Modeling of Battery Parameters and Behavior, (Master's Thesis). Retrieved from: http://repository.bilkent.edu.tr/ handle/11693/33537.

19. A. Aballe, M. Bethencourt, F. J. Botana, and M. Marcos, "Using wavelets transform in the analysis of electrochemical noise data," Electrochim. Acta., 44, 4805 (1999).

20. Y. Hoshi, N. Yakabe, K. Isobe, T. Saito, I. Shitanda, and M. Itagaki, "Wavelet transformation to determine impedance spectra of lithium-ion rechargeable battery," $J$. Power Sources., 315, 351 (2016).

21. Y. Hou, C. Aldrich, K. Lepkova, L. L. Machuca, and B. Kinsella, "Monitoring of carbon steel corrosion by use of electrochemical noise and recurrence quantification analysis," Corros. Sci., 112, 63 (2016).

22. P. R. Roberge, "Analysis of electrochemical noise by the stochastic process detector method," Corrosion. 50, 502 (1994).

23. J. D. Hunter, "Matplotlib: A 2D graphics environment," Comput. Sci. Eng., 9, 99 (2007).

24. S. Van Der Walt, S. C. Colbert, and G. Varoquaux, "The NumPy array: A structure for efficient numerical computation," Comput. Sci. Eng., 13, 22 (2011).

25. J. A. Motchenbacher and C. D. Connelly, Low-Noise Electronic System Design, p. 299, John Wiley \& Sons, New York (1993)

26. S. Piller, M. Perrin, and A. Jossen, "Methods for state-of-charge determination and their applications," J. Power Sources., 96, 113 (2001). 